\title{
Comparative genomic analysis of Enterococcus faecalis: insights into their environmental adaptations
}

\author{
Qiuwen He ${ }^{1,2}$, Qiangchuan Hou ${ }^{1,2}$, Yanjie Wang ${ }^{1,2}$, Jing Li ${ }^{1,2}$, Weicheng Li ${ }^{1,2}$, Lai-Yu Kwok ${ }^{1,2}$, Zhihong Sun ${ }^{1,2}$,
} Heping Zhang ${ }^{1,2}$ and Zhi Zhong ${ }^{1,2^{*}}$ (D)

\begin{abstract}
Background: Enterococcus faecalis is widely studied as a common gut commensal and a nosocomial pathogen. In fact, Enterococcus faecalis is ubiquitous in nature, and it has been isolated from various niches, including the gastrointestinal tract, faeces, blood, urine, water, and fermented foods (such as dairy products). In order to elucidate the role of habitat in shaping the genome of Enterococcus faecalis, we performed a comparative genomic analysis of 78 strains of various origins.
\end{abstract}

Results: Although no correlation was found between the strain isolation habitat and the phylogeny of Enterococcus faecalis from our whole genome-based phylogenetic analysis, our results revealed some environment-associated features in the analysed Enterococcus faecalis genomes. Significant differences were found in the genome size and the number of predicted open reading frames (ORFs) between strains originated from different environments. In general, strains from water sources had the smallest genome size and the least number of predicted ORFs. We also identified 293 environment-specific genes, some of which might link to the adaptive strategies for survival in particular environments. In addition, the number of antibiotic resistance genes was significantly different between strains isolated from dairy products, water, and blood. Strains isolated from blood had the largest number of antibiotic resistance genes.

Conclusion: These findings improve our understanding of the role of habitat in shaping the genomes of Enterococcus faecalis.

Keywords: Enterococcus faecalis, Genome, Environment, Antibiotic resistance gene, Phylogeny

\section{Background}

Enterococcus faecalis (E. faecalis) is a Gram-positive coccoid bacterium occurring singly, in pairs, in short chains, or in groups [1]; and it is the most common species within the genus Enterococcus. Many E. faecalis strains are associated with infections, including urinary tract infection, bacteraemia, endocarditis, neonatal infection, and infection of the central nervous system [2, 3]. Some $E$. faecalis strains have developed resistance to several

\footnotetext{
* Correspondence: imu150zhongzhi@163.com

${ }^{1}$ Key Laboratory of Dairy Biotechnology and Engineering, Ministry of Education P.R. C, Inner Mongolia Agricultural University, Huhhot 010018, People's Republic of China

${ }^{2}$ Key Laboratory of Dairy Products Processing, Ministry of Agriculture P.R.C, Inner Mongolia Agricultural University, Huhhot 010018, People's Republic of China
}

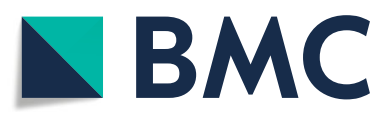

(c) The Author(s). 2018 Open Access This article is distributed under the terms of the Creative Commons Attribution 4.0 International License (http://creativecommons.org/licenses/by/4.0/), which permits unrestricted use, distribution, and reproduction in any medium, provided you give appropriate credit to the original author(s) and the source, provide a link to the Creative Commons license, and indicate if changes were made. The Creative Commons Public Domain Dedication waiver (http://creativecommons.org/publicdomain/zero/1.0/) applies to the data made available in this article, unless otherwise stated. of defence against a wide range of multi-resistant Grampositive pathogens [4]. The first vancomycin-resistant clinical strain of E. faecalis was reported in 1989 in the United States [5]. At present, E. faecalis is emerging as an important cause of hospital acquired infection and multidrug resistance [6]. For these reasons, E. faecalis is not generally regarded as safe (GRAS) [7].

Enterococcus faecalis is ubiquitous in nature and has been isolated from many different niches. The gastrointestinal (GI) tract of humans and animals is commonly considered as the primary habitat of E. faecalis, where it occurs as a commensal $[6,8,9]$. In addition, the blood and urine specimens of humans and animals are also major sources of E. faecalis [6]. As a species of lactic 
acid bacteria, E. faecalis is widely used in the production of fermented foods, particularly fermented dairy products. In recent years, numerous $E$. faecalis strains have been recovered from traditional dairy products [10-13]. Furthermore, water and soil are also common habitats of E. faecalis [14-16]. Considering the primary habitat of E. faecalis as the GI tract but meanwhile its wide distribution across numerous other niches makes it interesting to understand the relationship between enteric and extra-enteric E. faecalis strains. A key question is whether the extra-enteric E. faecalis strains are a product of faecal pollution or if they exist as independent lineages. Another intriguing aspect is how E. faecalis survives in and adapts to the highly diverse environments. Nowadays microbial genome sequencing provides an opportunity to answer these questions.

The complete genome of $E$. faecalis V583, one of the first reported vancomycin-resistant strains, was published in 2003 [17]. Subsequently, the genome sequences of 28 enterococcal strains (including $18 \mathrm{E}$. faecalis strains) were comparatively analysed to identify distinctive genetic traits and biochemical functions between lineages of clinical and environmental importance [18]. In 2014, the genomes of 38 E. faecalis strains were analysed to distinguish clinical from nonclinical strains [19]. In 2016, Raven et al. sequenced the whole genomes of three epidemic lineages isolated in the UK, analysed the genome-level data of altogether 168 E. faecalis strains, and described the evolution of vancomycin resistance within this strain collection [20]. As more and more genomes of $E$. faecalis from different habitats are available, we are now in a better position to understand the molecular basis of their environmental adaptation using comparative genomic analysis.

In this study, a total of 78 genomes of E. faecalis (including 15 genomes sequenced in this work and 63 genomes retrieved from the Genbank database) were subjected to comparative genomic analysis. These strains were isolated from faeces, blood, urine, dairy products, and water. We believe that results from this comparative genomic analysis can provide the insight necessary to understand the genetic relationships between these $E$. faecalis strains and the adaptive mechanisms that have evolved to allow them to occupy different niches.

\section{Methods}

\section{Bacterial strains}

A total of 78 genomes of $E$. faecalis were subjected to comparative genomic analysis. Among the 78 strains, 15 strains were collected from China, Russia, and Mongolia by our laboratory. These strains were isolated from naturally fermented dairy products and their genomes were sequenced in this study (Additional file 1). The other 63 genomes of $E$. faecalis were retrieved from the
Genbank database (Additional file 1). To ensure meaningful comparison between genomes and to analyse how the isolation habitat affected the genome characteristics, more than 400 genome sequence records of $E$. faecalis in the Genbank database were scanned. Only 63 genomes (including five complete genomes) were selected for this study based on a clear documentation of the strain isolation source and a high sequencing quality. The 63 strains were isolated from blood (20 strains), faeces (16 strains), urine (10 strains), dairy products (3 strains), water (11 strains), oral cavity (1 strain), and multiple sites (2 strains) (Additional file 1).

\section{DNA extraction}

Strains were cultured under anaerobic conditions in Man Rogosa and Sharpe (MRS) broth at $37{ }^{\circ} \mathrm{C}$. DNA was extracted from each strain using a bacterial DNA extraction kit (OMEGA D3350-02) according to the manufacturer's instructions. Genomic DNA was quantified using a TBS-380 fluorometer (Turner BioSystems Inc., Sunnyvale, CA). Only high-quality DNA samples $(\mathrm{OD} 260 / 280=1.8 \sim 2.0,>6 \mu \mathrm{g})$ were used to construct fragment libraries (200 to $300 \mathrm{bp}$ ).

\section{Sequencing, assembly, coding sequence (CDS) prediction, and annotation}

The whole-genome sequencing was done using the Illumina MiSeq platform (Illumina Inc., U.S.A) by generating $2 \times 150$ bp paired-end libraries using the Nextera DNA Sample Preparation Kit (Illumina Inc., U.S.A) following the manufacturer's instructions. On average, $625 \mathrm{Mb}$ of high-quality data were generated for each strain, corresponding to 176 - to 247 -fold sequencing depth (Additional file 1).

The paired-end reads were first assembled de novo using SOAPdenovo v1.06 [21]. Local inner gaps were filled and single base errors were corrected using the software GapCloser (http://sourceforge.net/projects/soapdenovo2/ files/GapCloser/). Coding sequences were predicted for each sequenced genome using Glimmer v3.02 [22]. Functional annotation of predicted open reading frames (ORFs) was achieved using RAST 2.0 [23] and COG database [24]. The individual genome assemblies of the 15 strains generated in this work were deposited in the National Center for Biotechnology Information under the accession numbers of MSQG00000000 to MSQU00000000 (Additional file 1).

\section{Construction of core- and pan-genomes}

The core- and pan-genomes of E. faecalis were constructed based on the families of homologous genes. The families of homologous genes for E. faecalis were computed using the SiLiX software [25]. Briefly, a pair of ORFs would be classified into the same gene family 
when their amino acid sequence identity value was above $80 \%$ and when the amino acid sequence alignment length spanned more than $80 \%$ of the longer ORF. All predicted ORFs of the 78 genomes were firstly grouped into their respective gene families before pan-genome construction. The pan-genome was constructed by counting the total number of non-redundant gene families within the complete dataset. The core-genome was constructed by counting the total number of gene families commonly shared by all genomes. The sequence of the longest ORF from each gene family was selected as the representative gene for functional annotation and phylogenetic reconstruction.

\section{Phylogenetic analysis}

A phylogenetic tree was constructed using the core genes of the 78 strains of $E$. faecalis. We first aligned the nucleotide sequences of the core genes using MUSCLE v3.8.31 [26], followed by removing the unreliable alignment regions and intragenic homologous recombination using Gblocks (http://molevol.cmima.csic.es/castresana/ Gblocks.html) and Gubbins (http://www.sanger.ac.uk/ science/tools/gubbins), respectively. A maximum likelihood tree was constructed based on the concatenated alignments using FastTree 2.1.8 [27] with 10,000 bootstrap iterations.

\section{Identification of environment-specific genes}

The subset of variable genes in the pan-genome was analysed to determine whether their distribution was significantly associated with the strain isolation niche (dairy, blood, faeces, urine, water, and oral cavity). If the frequency of a gene present in strains from one niche was much higher than the overall occurrence across all 78 strains, this gene was considered environment-specific. Scoary 1.6.16 (run with 1000 permutation replicates) was used to identify the spectrum of environment-specific genes, and the results were corrected for multiple testing. A $p$-value of less than 0.05 after Benjamini-Hochberg correction was considered significant [28].

\section{Identification of antibiotic resistance genes}

A BLAST search was performed with all predicted ORFs from the 78 strains against the Comprehensive Antibiotic Resistance Database (CARD; http://arpcard.mcmaster.ca) to identify potential antibiotic resistance genes (E-value of $<1 \mathrm{e}-15$ and sequence identity $>85 \%$ ) [29].

\section{Identification of virulence factors}

A BLAST search was performed with all predicted ORFs from the 78 strains against the Virulence Factor Database (VFDB) to identify genes encoding known virulence factors (E-value $<1 \mathrm{e}-15$ and sequence identity $>95 \%$ ) [30].

\section{Prophage identification}

Intact and incomplete prophage regions were identified through an integrated search with the annotation tool, PHASTER [31]. This involved genome-scale ORFs prediction and translation (via GLIMMER), protein identification (via BLAST matching and annotation by homology), phage sequence identification (via BLAST matching to a phage-specific sequence database), tRNA identification, and attachment site recognition. Only intact regions were analysed in-depth.

\section{Statistical analysis}

Data are presented as means \pm SEM. One-way ANOVA followed by Tukey's post-hoc test was used for statistical significance determination using SPSS Statistics 19 (IBM, Armonk, New York, USA). Significance was set at $p$-value $<0.05$.

\section{Results}

\section{General genomic characteristics of the species Enterococcus faecalis}

The analysed $E$. faecalis genomes had a low $\mathrm{G}+\mathrm{C}$ content ranging from 37.0 to $38.0 \%$ (Additional file 1). The average genome size was $2.94 \pm 0.15 \mathrm{Mb}$, with $2884 \pm$ 211 predicted ORFs (Additional file 1). Furthermore, there were significant differences in the genome size and the number of predicted ORFs between strains isolated from different sources (Fig. 1). On average, strains isolated from water sources had the smallest genome size and the lowest number of predicted ORFs, which were significantly different from those originated from blood $(P<0.01)$. In addition, strains from dairy products and blood differed significantly in their number of predicted ORFs $(p<0.01)$. There were no significant differences in the genome size nor the number of predicted ORFs between strains isolated from blood, faeces, and urine samples.

\section{The pan- and core-genome of the species Enterococcus faecalis}

The pan-genome of the 78 E. faecalis strains was composed of 10,573 gene families; and the pan-genome size grew continuously with the increase in newly deciphered genomes (Fig. 2a). In contrast, the size of the core-genome gradually stabilized and the increase in genome number had little influence on the core-genome size when the number of genomes reached 60-70 (Fig. 2b). The core gene set comprised 1361 genes, i.e. $47.2 \%$ of the number of predicted ORFs (2884 per genome), suggesting that over half of the predicted ORFs in each genome were dispensable.

Functional analysis of the representative genes in the pan- and core-genome was conducted using the COG database (Table 1). The core genes were mainly 

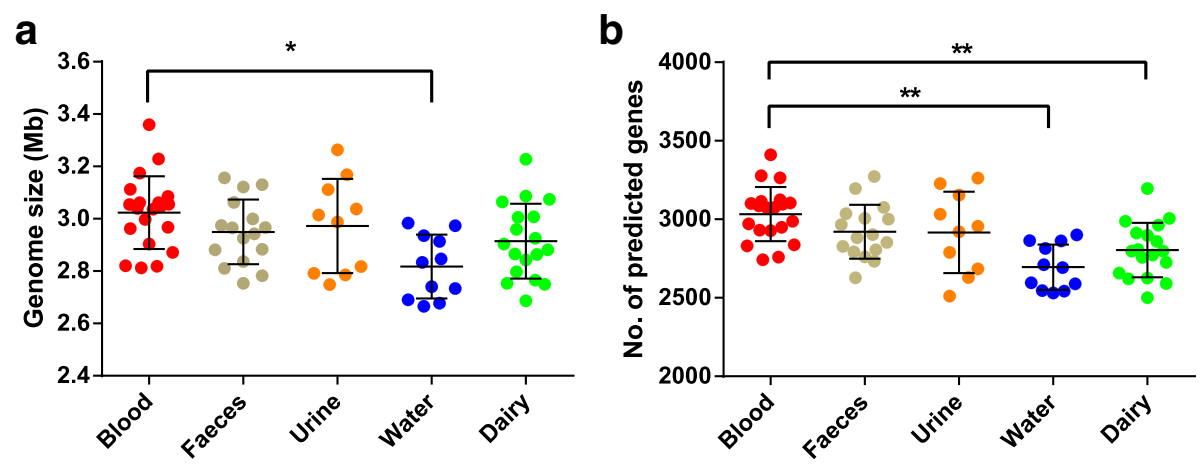

Fig. 1 Genome size (a) and number of predicted open reading frames (ORFs) (b) of Enterococcus faecalis strains isolated from different niches. An asterisk $\left(^{*}\right)$ indicates a $p$-value $<0.05$; double asterisks $\left(^{* *}\right)$ indicate a $p$-value $<0.01$ (one-way ANOVA test)

distributed in four categories, representing amino acid transport and metabolism; transcription; translation, ribosomal structure and biogenesis; and carbohydrate transport and metabolism. These genes together accounted for $33.4 \%$ of the core-genome. As a large proportion of genes were dispensable, pan-genome expansion happened in each functional category in varying degree compared with the core-genome (Fig. 3). The largest extent of expansion occurred in the group of genes involved in defence mechanisms (COG category [V]). Only 27 out of the 181 defence mechanisms-related genes within the pan-genome were core genes. The

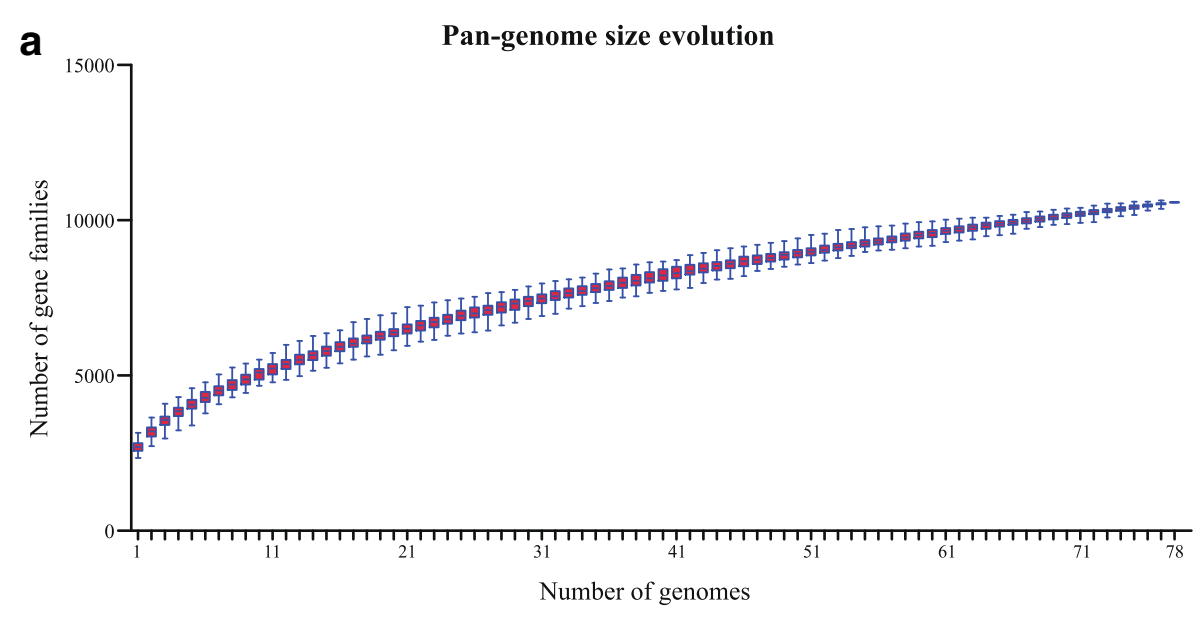

b

Core-genome size evolution

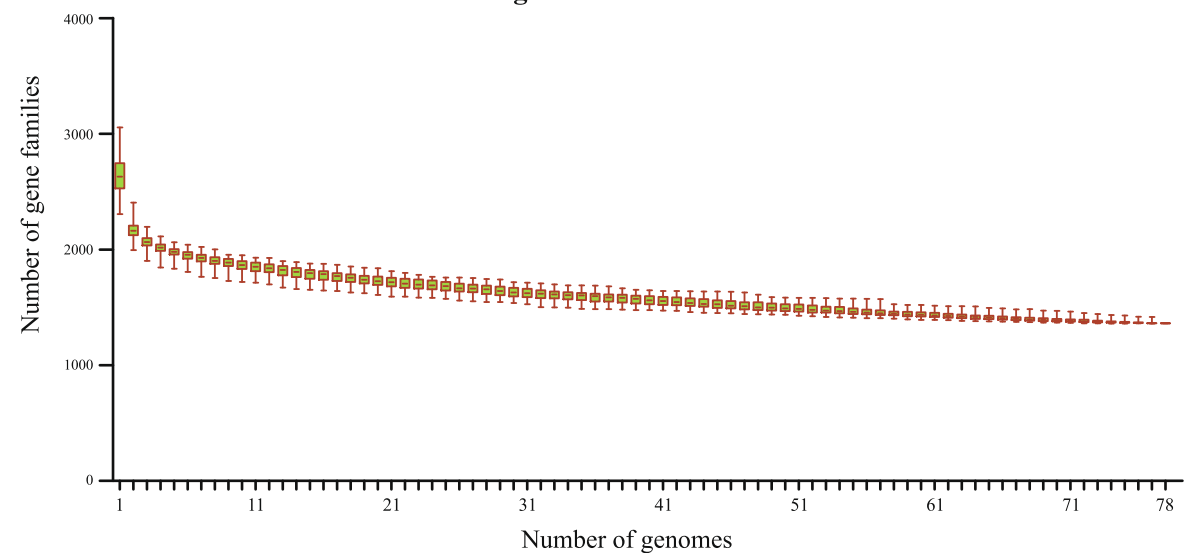

Fig. 2 Accumulation curves for pan-genomes (a) and core-genomes (b) of the species Enterococcus faecalis. The procedure was repeated 1000 times by randomly modifying the order of integration of genomes 
Table 1 Functional categories of core and pan genes in 78 Enterococcus faecalis genomes

\begin{tabular}{|c|c|c|c|}
\hline COG Functional category & $\begin{array}{l}\text { No. of core } \\
\text { genes }\end{array}$ & $\begin{array}{l}\text { No. of pan } \\
\text { genes }\end{array}$ & $\begin{array}{l}\text { Proportion of core genes } \\
\text { among pan genes (\%) }\end{array}$ \\
\hline Translation, ribosomal structure and biogenesis & 111 & 221 & 50.2 \\
\hline RNA processing and modification & 0 & 0 & 0.0 \\
\hline Transcription & 114 & 448 & 25.4 \\
\hline Replication, recombination and repair & 70 & 378 & 18.5 \\
\hline Chromatin structure and dynamics & 0 & 0 & 0.0 \\
\hline Cell cycle control, cell division, chromosome partitioning & 14 & 50 & 28.0 \\
\hline Nuclear structure & 0 & 0 & 0.0 \\
\hline Defense mechanisms & 27 & 181 & 14.9 \\
\hline Signal transduction mechanisms & 52 & 156 & 33.3 \\
\hline Cell wall/membrane/envelope biogenesis & 54 & 208 & 26.0 \\
\hline Cell motility & 5 & 18 & 27.8 \\
\hline Cytoskeleton & 0 & 1 & 0.0 \\
\hline Extracellular structures & 0 & 1 & 0.0 \\
\hline Intracellular trafficking, secretion, and vesicular transport & 15 & 59 & 25.4 \\
\hline Posttranslational modification, protein turnover, chaperones & 39 & 84 & 46.4 \\
\hline Energy production and conversion & 74 & 142 & 52.1 \\
\hline Carbohydrate transport and metabolism & 107 & 410 & 26.1 \\
\hline Amino acid transport and metabolism & 123 & 217 & 56.7 \\
\hline Nucleotide transport and metabolism & 61 & 99 & 61.6 \\
\hline Coenzyme transport and metabolism & 44 & 88 & 50.0 \\
\hline Lipid transport and metabolism & 32 & 74 & 43.2 \\
\hline Inorganic ion transport and metabolism & 86 & 164 & 52.4 \\
\hline Secondary metabolites biosynthesis, transport and catabolism & 19 & 50 & 38.0 \\
\hline General function prediction only & 172 & 439 & 39.2 \\
\hline Function unknown & 133 & 560 & 23.8 \\
\hline
\end{tabular}

smallest extent of expansion occurred in the COG categories of nucleotide transport and metabolism [F] and amino acid transport and metabolism [E]. More than half of the pan genes in these two functional categories were also core genes. These results suggest that E. faecalis possesses multiple defence mechanisms, while genes involved in nucleotide and amino acid transport and metabolism are generally more conserved.

\section{Phylogeny of the species Enterococcus faecalis}

To investigate the phylogenetic relationship between the 78 strains, the concatenated nucleotide sequence of the core genes of each strain was used to construct a phylogenetic tree. The 78 strains were divided into four branches on the phylogenetic tree (Fig. 4). The type strain (ATCC 19433) fell under branch A, which contained 19 strains, including seven dairy strains. Branch B comprised 22 strains. Branch $C$ was the smallest branch with only 16 strains. Branch D composed of 21 strains of which none was isolated from urine samples. Strains originated from different niches were evenly dispersed across the four branches, suggesting no correlation between strain isolation niche and strain phylogeny.

\section{Environment-specific genes}

Pan-GWAS analysis was performed with Scoary to identify genes that were present in strains associated with a particular environment [28]. A total of 293 environment-specific genes were identified, including 143,66 , and 84 genes that were specifically linked to strains isolated from blood, dairy, and water sources, respectively (Fig. 5). Most of the environment-specific genes encoded hypothetical proteins with unknown function. The environment-specific genes of known function were analysed using the COG database; and most of them were involved in carbohydrate transport and metabolism (Additional file 2).

Among the blood-specific genes, five genes were involved in galactose metabolism: dgoD encodes a galactonate dehydratase; PTS-Aga-EIID encodes the N-acetylgalactosamine-specific IID component of phosphotransferase system (PTS); PTS-Gat-EIIA, B, and 


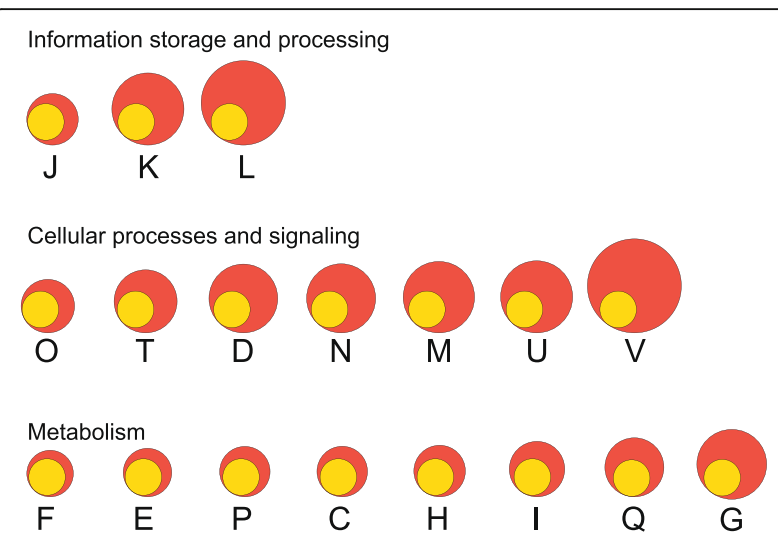

Fig. 3 Expansion of the pan-genome compared with the core-genome for each functional category. The number of core genes in each functional category was normalized (yellow circle). The magnification of the pan genes compared with the core genes in each functional category is shown by the red circle. The letter below the circle represents the functional category: [J] Translation, ribosomal structure and biogenesis; [K] Transcription; [L] Replication, recombination and repair; [O] Posttranslational modification, protein turnover, chaperones; [1] Signal transduction mechanisms; [D] Cell cycle control, cell division, chromosome partitioning; [N] Cell motility; [M] Cell wall/membrane/ envelope biogenesis; [U] Intracellular trafficking, secretion, and vesicular transport; [V] Defence mechanisms; [F] Nucleotide transport and metabolism; [E] Amino acid transport and metabolism; [P] Inorganic ion transport and metabolism; [C] Energy production and conversion; $[\mathrm{H}]$ Coenzyme transport and metabolism; [1] Lipid transport and metabolism; [Q] Secondary metabolites biosynthesis, transport and catabolism; [G] Carbohydrate transport and metabolism

$\mathrm{C}$ encode the galactitol-specific IIA, B, and C components of PTS system, respectively. Furthermore, panD encodes an aspartate 1-decarboxylase that involves in beta-alanine metabolism; eda encodes the 2-dehydro-3deoxyphosphogluconate aldolase / (4S)-4-hydroxy-2-oxoglutarate that involves in carbon metabolism; znuA encodes a zinc transport system substrate-binding protein.

Among the dairy-specific genes, the genes phnC, phnD, and phnE encode for the phosphonate transport system ATP-binding protein, phosphonate transport system substrate-binding protein, and phosphonate transport system permease protein, respectively. Three genes constitute the $\mathrm{ABC}$ transporter of phosphonate. PTS-Cel-EIIC and PTS-Lac-EIIC encode the cellobiosespecific IIC component and lactose-specific IIC component of the PTS system, respectively. PldB encodes a lysophospholipase that involves in glycerophospholipid metabolism. Dld encodes a D-lactate dehydrogenase that involves in pyruvate metabolism. ClpL encodes an ATP-dependent Clp protease ATP-binding subunit.

Among the 84 water-specific genes, 28 were present in all 11 strains isolated from water sources. Among these, cbiO encodes an ATP-binding protein, while cbiM and cbiQ both encode permease proteins. Three genes were involved in the cobalt and nickel transport system: znuC is a zinc transport system ATP-binding protein involved in zinc transport; the genes PTS-Man-EIIB and PTS-Man-EIIC encode mannose-specific IIB and IIC components of PTS system, respectively. DdhP encodes an alcohol dehydrogenase involved in tyrosine metabolism and fatty acid degradation. PcaC encodes a 4-carboxymuconolactone decarboxylase involved in benzoate degradation. RP-L33 encodes the large subunit ribosomal protein L33.

\section{Antibiotic resistance genes}

Potential antibiotic resistance genes were detected by blasting the $78 \mathrm{E}$. faecalis genomes against the CARD database (Additional file 3). There were on average 7.5 antibiotic resistance genes in each genome. The number of antibiotic resistance genes varied greatly between strains. Four blood originated strains (DAPTO 516, DAPTO 512, S613, and R712) and one faecal strain (TX0104) had the highest number of antibiotic resistance genes; each of them possessed 18 antibiotic resistance genes. Thirty-four strains contained the fewest antibiotic resistance genes, with only five antibiotic resistance genes per genome.

Based on the average number of antibiotic resistance genes, the 78 investigated strains could be divided into three classes. The first class included strains isolated from blood with the largest number of antibiotic resistance genes (10.4 antibiotic resistance genes per strain). The second class included strains isolated from faeces (7.4 antibiotic resistance genes per strain) and urine (8.1 antibiotic resistance genes per strain). The last class included strains from dairy (5.3 antibiotic resistance genes per strain) and water sources (5.2 antibiotic resistance genes per strain) with the fewest antibiotic resistance genes. Furthermore, the number of antibiotic resistance genes present in the blood originated strains was significantly different from those isolated from dairy and water sources $(p<0.01)$ (Fig. 6).

Although the distribution of antibiotic resistance genes varied greatly between strains, five antibiotic resistance genes were commonly present in all 78 strains. Most of these antibiotic resistance genes were involved in efflux-mediated resistance to antibiotics. LsaA encodes an $\mathrm{ABC}$ efflux pump. It confers resistance to clindamycin, quinupristin-dalfopristin, and dalfopristin. EmeA encodes a multidrug efflux pump [32]. EfrA and efrB encode two subunits of the EfrAB efflux pump, which are related to the drug resistance in both $E$. faecalis and $E$. faecium. In addition to the efflux-mediated resistance genes, dfrE encodes a dihydrofolate reductase that confers resistance to trimethoprim.

Moreover, we found two types of vancomycin-resistance gene clusters among the 78 strains: vanA-type and vanB-type. The vanA-type cluster is $8.1 \mathrm{~kb}$, containing six 


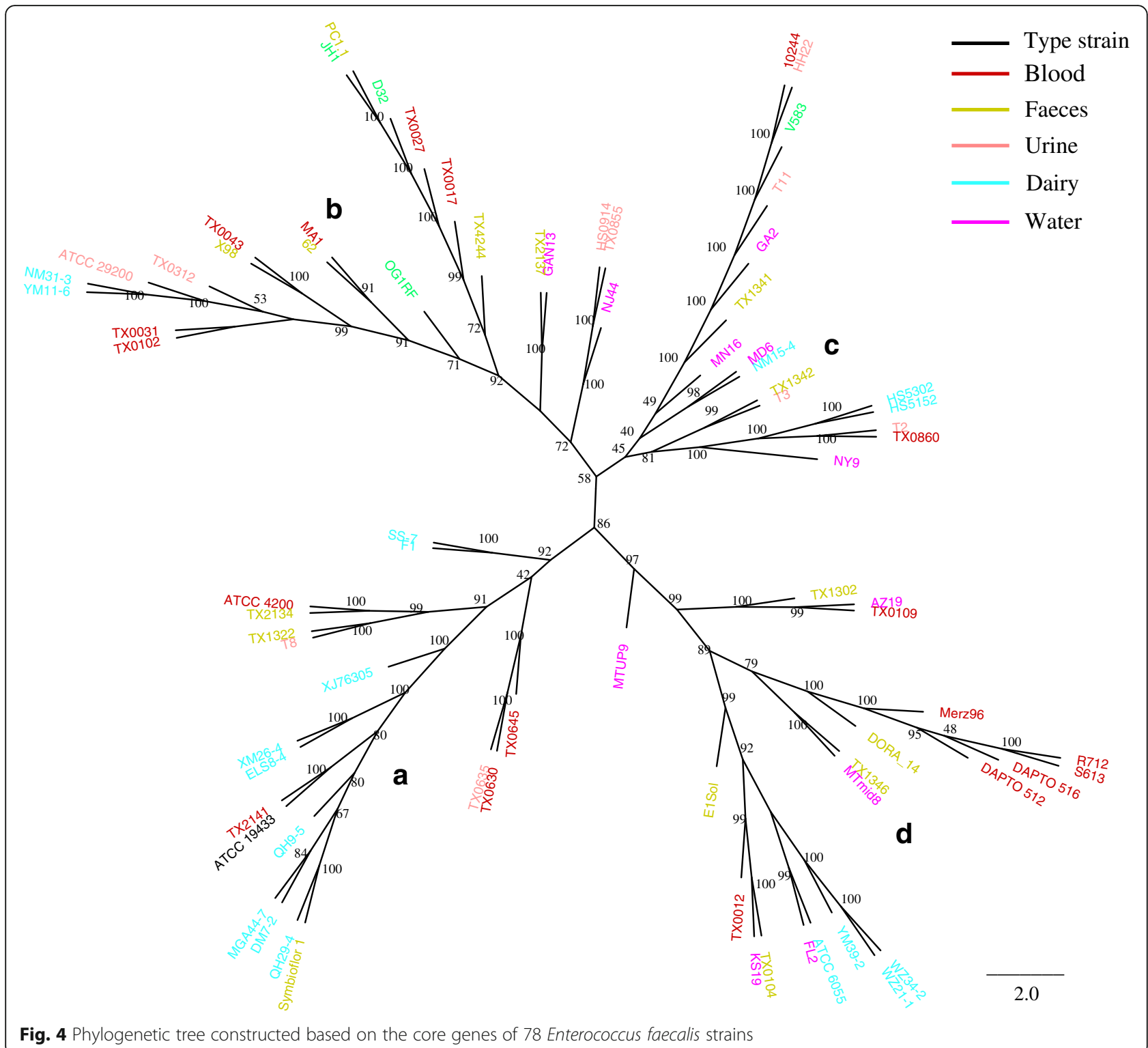

vancomycin-resistant genes: vanZA, vanYA, vanXA, vanHA, vanSA, and vanRA [33]. The vanA-type cluster was only found in four strains isolated from blood (DAPTO 512, DAPTO 516, R712, and S613). The vanB-type is $6.4 \mathrm{~kb}$, containing six vancomycin-resistant genes: van $\mathrm{RB}, \operatorname{van} \mathrm{SB}, \operatorname{van} \mathrm{YB}, \operatorname{van} \mathrm{WB}$, van $\mathrm{HB}$, and $\operatorname{vanXB}$ [33]. The vanB-type cluster was exclusively identified in two strains of blood origin (V583 and Merz96) and one faecal strain (TX0104).

\section{Virulence factors}

Genes coding for known virulence factors were identified by blasting the $78 \mathrm{E}$. faecalis genomes against the VFDB database. Sixty putative virulence factors (23.8 per genome) were detected within the $78 \mathrm{E}$. faecalis genomes (Additional file 4). The number of virulence factors varied greatly between strains. The blood originated strain, V583, had the highest number of virulence factors (52 within the genome), contrasting to the strains E1Sol, TX1322 (both isolated from faeces samples), and T3 (isolated from a urine specimen) that carried only a low number of nine virulence factors per strain. Furthermore, no significant difference existed in the number and functions of virulence factors between strains of different isolation sources.

In summary, the virulence factors of E. faecalis were mainly involved in adherence, antiphagocytosis, biofilm formation, quorum sensing system, production of exoenzymes and toxins. The most common virulence factors were responsible for adherence (24 out of the 60 putative virulence factors), including ebpA/B/C (encode three Ebp pili subunits that facilitate bacterial adherence to host 


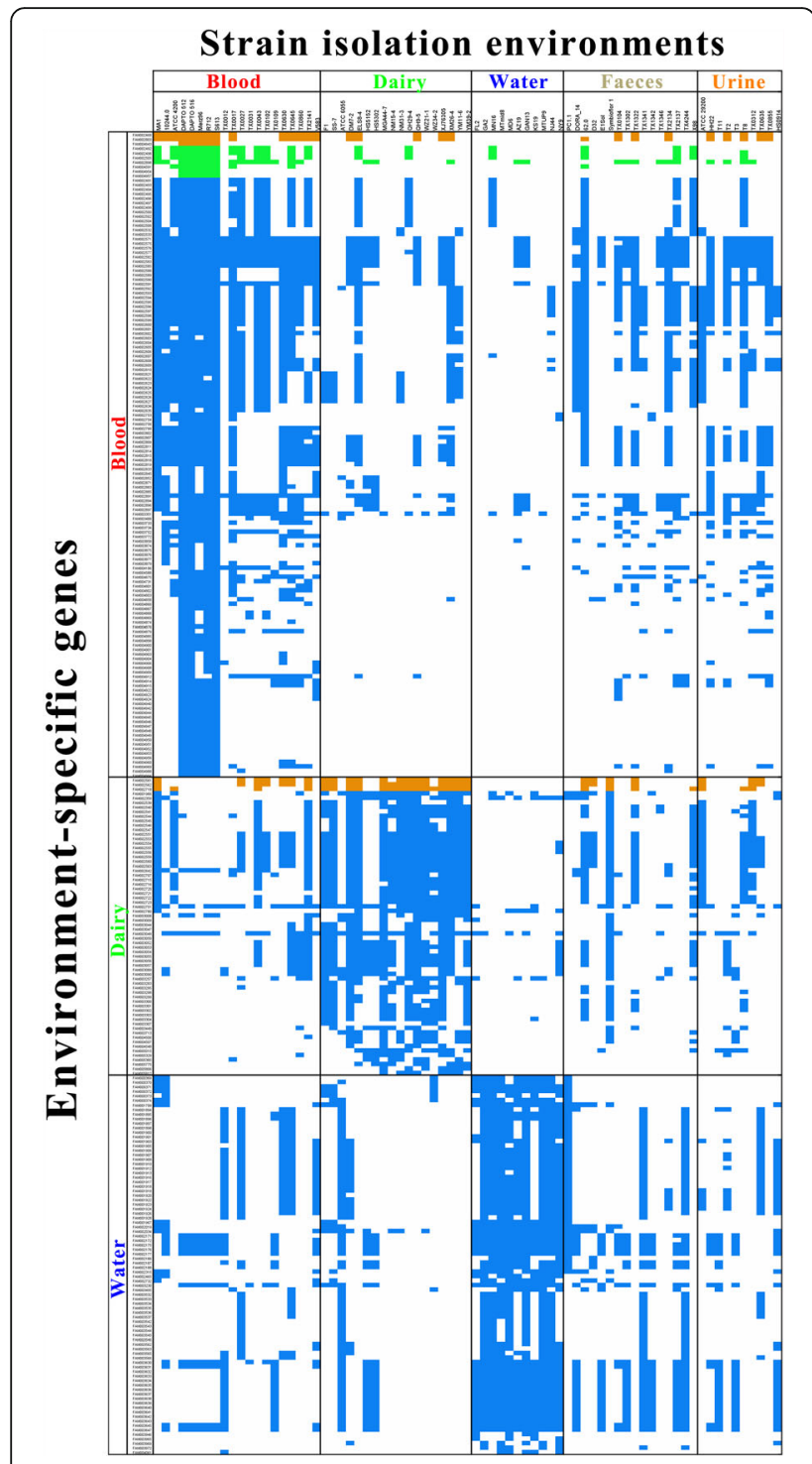

Fig. 5 Heatmap of environment-specific genes. The blue dots represent genes present in specific strains. Genes annotated as "transposase" and "phage protein" are highlighted in yellow and green, respectively

extracellular matrix proteins), ace (encodes a collagen adhesin), and asa1 (encodes aggregation substance). Another common putative virulence factor present in $E$. faecalis was the csp operon. The csp operon consists of 11 ORFs (i.e. cpsA to cpsK), encoding an antiphagocytosis factor that facilitates bacterial evasion of the host immune system. Moreover, some members of the cytolysin (cyl) operon were detected in the E. faecalis genomes. Normally, the cyl operon comprises eight genes, i.e. cylA/B/I/ $\mathrm{L} / \mathrm{M} / \mathrm{R} 1 / \mathrm{R} 2 / \mathrm{S}$. However, only four genes, cylA/B/I/M, were detected in five of the studied strains, including two urine isolated strains (T2 and T8), two dairy strains (SS-7 and F1), and one blood originated strain (Merz96).

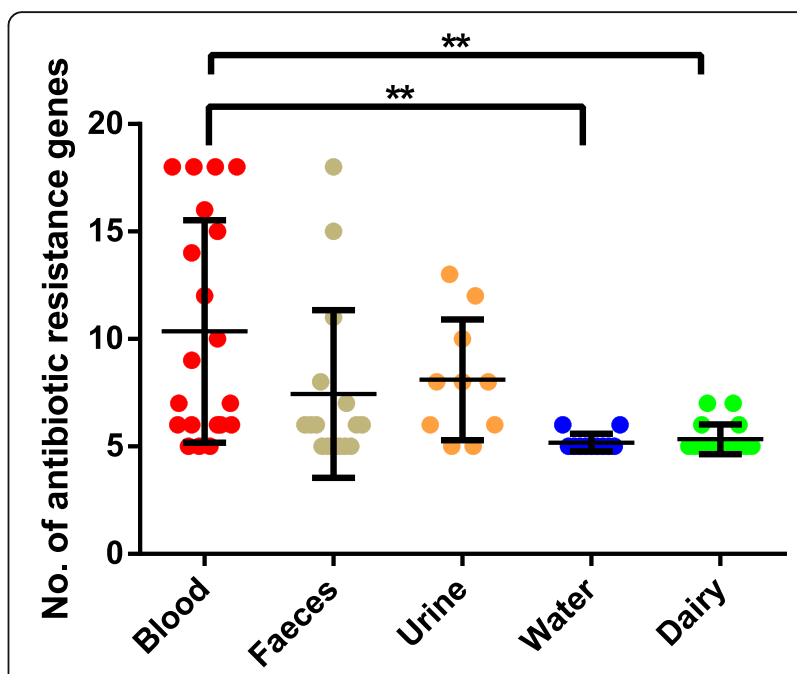

Fig. 6 The number of antibiotic resistance genes in strains isolated from different niches. Double asterisks $\left(^{* *}\right)$ indicate a $p$-value $<0.01$ (one-way ANOVA test)

\section{Prophage sequences}

A total of 116 intact prophages were identified (Additional file 5). Not all investigated E. faecalis genomes contained intact prophages (detected only in 65 out of 78 genomes). No apparent correlation was found between the isolation source and occurrence of intact prophage sequences. The 13 strains that contained no intact prophage were originated from all types of environments, including four dairy strains (HS5152, HS5302, WZ34-2, XJ76305), three water originated strains (GA2, KS19, MTmid8), two faecal strains (TX0104, TX4244), two urine isolated strains (HH22, T11), one blood isolated strain (TX0031), and one oral strain (OG1RF).

The strain TX0645 (isolated from blood), ATCC6055 (isolated from dairy), and X98 (isolated from faeces) contained the highest number of intact prophages (four per genome). Apart from some common enterococcal prophages (e.g. phiEf11, phiFL4A, SANTOR1, and vB), prophages associated with other bacterial genera (including Lactobacillus, Lactococcus, Listeria, Bacillus, Clostridium, Staphylococcus, Streptococcus, and Weissella) were also detected. Such results together suggest that prophages are extensively propagated intra- and inter-species; and their spread is independent from the isolation source.

\section{Discussion}

In this study, we performed a comparative genomic analysis of $78 \mathrm{E}$. faecalis strains (15 genomes sequenced by this work plus 63 genome sequences retrieved from the Genbank database). These strains were originated from a wide range of sources, including blood, faeces, urine, dairy products, water, and oral cavity. Our work has taken the advantage of the wide ecological niches of 
these strains to elucidate the role of habitat in shaping the genome characteristics of the E. faecalis species.

The pan-genome of the $78 \mathrm{E}$. faecalis strains comprised 10,573 gene families, of which 1361 genes were conserved across all studied strains. This corroborates the results derived from the previous analysis of 168 strains [20]. Comparing with the core-genome, the biggest pan-genome expansion occurred in the subset of defence mechanisms-related genes. Only 14.9\% (27 out of 181) of the defence mechanisms-related genes were core genes. The 27 core genes mainly encode the ATP-binding cassette $(\mathrm{ABC})$ transport system permease and $A B C$ subfamily $B$, which confer multi-drug resistance to the bacteria [34]. The variable gene portion mainly encodes restriction endonucleases of type I restriction modification system, which function to defend against invading viruses, such as bacteriophage $[35,36]$. The type I restriction modification system consists of the $\mathrm{R}, \mathrm{M}$, and $\mathrm{S}$ subunits. Our study identified mainly the $\mathrm{S}$ subunit that is responsible for determining the specificity of the DNA-binding site recognition during DNA cleavage and modification of the enterococcal genomes $[37,38]$. The high variability of this spectrum of genes suggests the existence of multiple defence mechanisms to protect the cells from viral invasion. Furthermore, the defence mechanisms of E. faecalis do not seem to be associated with the strain isolation habitat, as no environment-specific genes were identified within this functional category.

We then performed a phylogenetic reconstruction using the core-genome of all $78 \mathrm{E}$. faecalis strains. The results suggested no correlation between the strain isolation habitat and phylogeny, which corroborates the inference drawn by a previous study [39]. However, our in-depth analysis of the functional genomes did reveal environment-specific adaptation in E. faecium strains originated from dairy products, water, and blood.

Milk is a lactose-rich environment. Some of the identified dairy-specific genes seem to help the dairy enterococci utilize the lactose present in the milk environment. For example, the dairy-specific gene dld encodes D-lactate dehydrogenase, which has previously been found in plasmids from Lactococcus lactis and Lactobacillus delbrueckii subsp. bulgaricus. D-lactate dehydrogenase may involve in D-lactate utilization under aerobic conditions [40]. During milk fermentation, D-lactate utilization might result in $\mathrm{pH}$ reduction or the sugar could be converted to acetate accompanied with ATP production; both activities would enhance bacterial survival in dairy products. Siezen et al. suggested that the dld gene might have been acquired from Gram-negative bacteria by horizontal transfer, as the best homologues (about 50\% sequence identity and the same size) are found exclusively in Gram-negative bacteria such as Escherichia coli and Shigella spp. but not in any Gram-positive bacteria [40].

Another set of dairy-specific genes was lacE, lacF, and lacG. In Gram-positive bacteria, lactose is internalized by the phosphoenolpyruvate-dependent PTS, which consists of the lactose-specific IIBC and IIA components (encoded by lacE and lacF, respectively), yielding lactose-6-phosphate. Lactose-6-phosphate is then hydrolyzed to glucose and galactose-6-phosphate by a cytoplasmic phospho- $\beta$-galactosidase (encoded by lacG) [41] .

Among the water-specific genes, cbiM, cbiO, and cbiQ encode part of the cobalt and nickel transport system. Furthermore, metal uptake operons were widely distributed in all 11 strains from water sources. This result is in line with the study of [9]. The transition metals, nickel and cobalt, are essential cofactors for many prokaryotic enzymes involved in a variety of metabolic processes [42]. The mean concentration of nickel in freshwater environments is about $10 \mu \mathrm{g} / \mathrm{L}$, which is much lower than other environments [14]. Thus, a high-affinity nickel uptake system may be beneficial to the survival of the water-dwelling $E$. faecalis strains.

Interestingly, some environment-specific genes do not seem to have any adaptive relationship with the isolation habitat. For example, the dairy-specific genes phnC, phnD, and phnE constitute the integrated $A B C$ transporter of phosphonates, which are quite common among many organisms, ranging from bacteria, fungi, molluscs, insects, plants, and animals. These three genes were identified in 15 of the 18 dairy-associated E. faecalis strains. However, there is no indication as to why the integrated $\mathrm{ABC}$ transporter of phosphonates should be specifically enriched in dairy strains. Nevertheless, the precise role of natural phosphonates is still poorly understood. A similar situation occurred with the blood-specific genes PTS-Gat-EIIA, PTS-Gat-EIIB, and PTS-Gat-EIIC, which constitute the PTS system involved in galactitol metabolism. To our knowledge, plasma galactitol is only an important parameter for the assessment of steady-state galactose metabolism in galactosaemia [43, 44]. A large number of environment-specific genes are hypothetical genes with unknown function. These genes may encode additional environment-associated functions and require further research.

Another interesting phenomenon observed in this work was the differences in the antibiotic resistance gene profile between strains isolated from various niches. Strains from blood sources had significantly more antibiotic resistance genes than those from dairy and water sources. This is in line with the results of Raven et al. that reported an enrichment of antibiotic resistance genes in epidemic lineages [20]. The pressure of natural selection may play a key role in the uneven distribution of antibiotic resistance genes in this case. For example, 
the tetM gene codes a ribosomal protection protein that confers tetracycline resistance. This gene is known to associate with or even encoded by transposable DNA elements, and its horizontal transfer between bacterial species has been documented [45]. Our study showed that tetM was present mostly in strains isolated from blood (15 out of 20) and occurred in much lower frequency in strains originated from dairy (4 out of 18) and water (2 out of 11) sources. Tetracyclines have a broad spectrum of antibiotic action and are commonly used in treating bacterial infections; thus, strains from blood sources are more likely to be exposed to the bacteriostatic activity of tetracyclines and that strains lacking tetracycline resistance genes would be outcompeted rapidly. This explains the high proportion of tetM-positive blood originated strains. In contrast, strains from dairy and water sources would not need tetracycline resistance genes for survival because of the much lower environmental selection pressure of tetracycline; thus, these genes were not maintained in the bacterial genomes.

Five antibiotic resistance genes were found among the 78 strains, although the number of antibiotic resistance genes found in each strain varied greatly. Most of these antibiotic resistance genes were involved in efflux-mediated resistance to antibiotics. Efflux was first described as a mechanism of resistance to tetracycline. In recent years, numerous plasmid- and chromosome-encoded efflux mechanisms have been described in various microorganisms [46]. The fact that the efflux-mediated resistance is coded by core genes implicates that it is an important intrinsic resistance mechanism of E. faecalis.

Previous studies have characterised six types of vancomycin resistance in enterococci [33]. Our study found two types of vancomycin-resistant gene clusters among the 78 strains, i.e. vanA and vanB type clusters. VanA is the most frequently encountered type of glycopeptide resistance in enterococci; strains that have acquired vanA are resistant to high levels of vancomycin [33]. Raven et al. found that nearly all vancomycin-resistant $E$. faecalis carried vanA cluster [20]. Our study identified the vanA type gene cluster only in four blood originated strains. It is interesting to note that these four strains were most closely related genetically (Fig. 4). This may indicate that the common ancestor of these four strains had acquired the vanA type resistant gene cluster before being internalized in this lineage. The organization and functionality of the vanB type cluster is similar to that of vanA, but the resistant levels are variable [33]. Among the isolates we studied, the three vanB-positive strains were isolated from blood and faeces. Our results together show that the blood isolated strains had more vancomycin-resistant genes than those from other sources.
There are some limitations of this work. The strain collection used was relatively small for genome-wide study, which might lead to bias of some results, such as the identified environment-specific genes. Moreover, in addition to isolation habitat, other factors, e.g. geographic origin and year of collection, might also involve in shaping the bacterial genomes. However, due to incomplete strain documentation in some of the retrieved records, our work could not cover these aspects.

\section{Conclusions}

In summary, although there was no correlation between the strain isolation source and phylogeny, our results did demonstrate that habitat was involved in shaping the $E$. faecalis genomes. There were significant differences in the genome size and number of predicted ORFs between strains isolated from different habitats. Furthermore, some environment-specific genes were found in strains isolated from dairy, blood, and water sources; and some of these genes might improve the adaptive capacity of the strains to survive in their dwelling environment. In addition, strains from blood had the largest number of antibiotic resistance genes. All these findings suggest that the natural habitat where the strain was recovered is effective in shaping the E. faecalis genomes.

\section{Additional files}

Additional file 1: List of the Enterococcus faecalis strains analyzed in this study. The strain and genome information is listed in this table. (XLS 17 kb)

Additional file 2: List of environment-specific genes in the investigated Enterococcus faecalis strains. A total of 293 environment-specific genes were identified, including 143, 66, and 84 genes that were specifically linked to strains isolated from blood, dairy, and water sources, respectively. The function of environment-specific genes was annotated based on the COG, KEGG, and $\mathrm{nr}$ databases. (XLS $69 \mathrm{~kb}$ )

Additional file 3: Antibiotic resistance genes found in the 78 Enterococcus faecalis strains. Potential antibiotic resistance genes were detected by blasting the $78 \mathrm{E}$. faecalis genomes against the CARD database (E-value of <1e-15 and sequence identity > 85\%). (XLS 94 kb)

Additional file 4: Virulence factors found in the 78 Enterococcus faecalis strains. Genes coding for known virulence factors were identified by blasting the $78 \mathrm{E}$. faecalis genomes against the VFDB database (E-value $<1 \mathrm{e}-15$ and sequence identity $>95 \%$ ). Sixty putative virulence factors (23.8 per genome) were detected within the 78 E. faecalis genomes. (XLS $237 \mathrm{~kb}$ )

Additional file 5: Distribution of the intact prophage regions among the Enterococcus faecalis strains. Intact prophage regions were identified through PHASTER. A total of 116 intact prophages were identified. (XLS $45 \mathrm{~kb}$ )

\section{Abbreviations}

ABC: ATP-binding cassette; CARD: Comprehensive Antibiotic Resistance Database; E. faecalis: Enterococcus faecalis; Gl: Gastrointestinal;

GRAS: Generally regarded as safe; VFDB: Virulence Factor Database

Acknowledgements

We would like to thank Dr. Judy Pell for manuscript revision.

\section{Funding}

This research was supported by the National Natural Science Foundations of China (Grant No. 31601451 and 31622043). The funding bodies had no role 
in the design of the study, collection and analysis of data, interpretation of results, or in writing the manuscript.

\section{Availability of data and materials}

The individual genome assemblies of the 15 strains sequenced in this work were deposited in the National Center for Biotechnology Information under the accession numbers of MSQG00000000 to MSQU00000000 (https:// www.ncbi.nlm.nih.gov/assembly/) (Additional file 1).

\section{Authors' contributions}

$\mathrm{ZZ}, \mathrm{HZ}$, and $\mathrm{ZS}$ designed the study. ZZ, QWH, QCH, YW, JL, and WL performed the de novo assembly and comparative genomics analyses. $\mathrm{QWH}$ LYK, QCH, ZS, and ZZ wrote the manuscript. All authors read and approved the final manuscript

\section{Ethics approval and consent to participate}

There was no animal experimentation undertaken in this study.

\section{Consent for publication}

Not applicable.

\section{Competing interests}

The authors declare that they have no competing interests.

\section{Publisher's Note}

Springer Nature remains neutral with regard to jurisdictional claims in published maps and institutional affiliations.

Received: 16 November 2017 Accepted: 19 June 2018

Published online: 11 July 2018

\section{References}

1. Holzapfel WH, Wood BJB. Lactic Acid Bacteria: Biodiversity and Taxonomy. 1st ed. Chichester: Wiley-Blackwell; 2014

2. Moellering RC Jr. Emergence of Enterococcus as a significant pathogen. Clin Infect Dis. 1992;14(6):1173-6.

3. O'Driscoll T. Crank CW. Vancomycin-resistant enterococcal infections: epidemiology, clinical manifestations, and optimal management. Infect Drug Resist. 2015:8:217-30.

4. Moscoso M, Domenech M, Garcia E. Vancomycin tolerance in Gram-positive cocci. Environ Microbiol Rep. 2011:3(6):640-50.

5. Sahm DF, Kissinger J, Gilmore MS, Murray PR, Mulder R, Solliday J, Clarke B. In vitro susceptibility studies of vancomycin-resistant Enterococcus faecalis. Antimicrob Agents Chemother. 1989;33(9):1588-91.

6. Lebreton F, Willems RJL, Gilmore MS. Enterococcus Diversity, Origins in Nature, and Gut Colonization. In: Gilmore MS, D. B. Clewell DB, Ike Y, and Shankar N, editors. Enterococci: From Commensals to Leading Causes of Drug Resistant Infection. Boston: Massachusetts Eye and Ear Infirmary; 2014. p. 5-64.

7. Ogier JC, Serror P. Safety assessment of dairy microorganisms: the Enterococcus genus. Int J Food Microbiol. 2008;126(3):291-301.

8. Lukasova J, Sustackova A. Enterococci and antibiotic resistance. Acta Vet Brno. 2003;72(2):315-23.

9. Barretto Penna AL, de Paula AT, Casarotti SN, Silva LF, Diamantino VR, Todorov SD. Overview of the functional lactic acid Bacteria in fermented milk products. In: Ravishankar VR, Jamuna $A B$, editors. Beneficial microbes in fermented and functional foods. Boca Raton: CRC Press; 2015. p. 113-48.

10. Chen X, Song YQ, Xu HY, Menghe BL, Zhang HP, Sun ZH. Genetic relationships among Enterococcus faecalis isolates from different sources as revealed by multilocus sequence typing. J Dairy Sci. 2015:98(8):5183-93.

11. Delgado S, Mayo B. Phenotypic and genetic diversity of Lactococcus lactis and enterococcus spp. strains isolated from northern Spain starter-free farmhouse cheeses. Int J Food Microbiol. 2004;90(3):309-19.

12. Silvetti T, Morandi S, Brasca M. Biopreservation potential of enterococcus faecalis isolated from Italian traditional raw milk cheeses. Cyta-J Food. 2014; 12(3):210-7.

13. Terzic-Vidojevic A, Veljovic K, Begovic J, Filipic B, Popovic D, Tolinacki M, et al. Diversity and antibiotic susceptibility of autochthonous dairy enterococci isolates: are they safe candidates for autochthonous starter cultures? Front Microbiol. 2015;6:954

14. Weigand MR, Ashbolt NJ, Konstantinidis KT, Santo Domingo JW. Genome sequencing reveals the environmental origin of enterococci and potential biomarkers for water quality monitoring. Environ Sci Technol. 2014;48(7): 3707-14.

15. Byappanahalli MN, Nevers MB, Korajkic A, Staley ZR, Harwood VJ. Enterococci in the environment. Microbiol Mol Biol Rev. 2012;76(4):685-706.

16. Veljović K, Popović N, Terzić Vidojević A, Tolinački M, Mihajlović S, Jovčić B, et al. Environmental waters as a source of antibiotic-resistant enterococcus species in Belgrade, Serbia. Environ Monit Assess. 2015;187(9):1-15.

17. Paulsen IT, Banerjei L, Myers GS, Nelson KE, Seshadri R, Read TD, Fouts DE, Eisen JA, Gill SR, Heidelberg JF, Tettelin H, Dodson RJ, Umayam L, Brinkac L, Beanan M, Daugherty S, DeBoy RT, Durkin S, Kolonay J, Madupu R, Nelson W, Vamathevan J, Tran B, Upton J, Hansen T, Shetty J, Khouri H, Utterback T, Radune D, Ketchum KA, Dougherty BA, Fraser CM. Role of mobile DNA in the evolution of vancomycin-resistant Enterococcus faecalis. Science. 2003; 299(5615):2071-4.

18. Palmer KL, Godfrey P, Griggs A, Kos VN, Zucker J, Desjardins C, Cerqueira G, Gevers D, Walker S, Wortman J, Feldgarden M, Haas B, Birren B, Gilmore MS. Comparative genomics of enterococci: variation in Enterococcus faecalis, clade structure in E. Faecium, and defining characteristics of E. Gallinarum and E. Casseliflavus. mBio. 2012;3(1):e00318-1.

19. Kim EB, Marco ML. Nonclinical and clinical Enterococcus faecium strains, but not enterococcus faecalis strains, have distinct structural and functional genomic features. Appl Environ Microbiol. 2014;80(1):154-65.

20. Raven KE, Reuter S, Gouliouris T, Reynolds R, Russell JE, Brown NM, Torok ME, Parkhill J, Peacock SJ. Genome-based characterization of hospitaladapted Enterococcus faecalis lineages. Nat Microbiol. 2016;1:15033.

21. Luo R, Liu B, Xie Y, Li Z, Huang W, Yuan J, et al. SOAPdenovo2: an empirically improved memory-efficient short-read de novo assembler. GigaScience. 2012;1(1):18.

22. Delcher AL, Bratke KA, Powers EC, Salzberg SL. Identifying bacterial genes and endosymbiont DNA with Glimmer. Bioinformatics. 2007:23(6):673-9.

23. Aziz RK, Bartels D, Best AA, DeJongh M, Disz T, Edwards RA, Formsma K, Gerdes S, Glass EM, Kubal M, Meyer F, Olsen GJ, Olson R, Osterman AL, Overbeek RA, McNeil LK, Paarmann D, Paczian T, Parrello B, Pusch GD, Reich C, Stevens R, Vassieva O, Vonstein V, Wilke A, Zagnitko O. The RAST server: rapid annotations using subsystems technology. BMC Genomics. 2008;9:75.

24. Tatusov RL, Fedorova ND, Jackson JD, Jacobs AR, Kiryutin B, Koonin EV, Krylov DM, Mazumder R, Mekhedov SL, Nikolskaya AN, Rao BS, Smirnov S, Sverdlov AV, Vasudevan S, Wolf YL, Yin JJ, Natale DA. The COG database: an updated version includes eukaryotes. BMC Bioinformatics. 2003;4:41.

25. Miele $\mathrm{V}$, Penel $\mathrm{S}$, Duret L. Ultra-fast sequence clustering from similarity networks with SiLiX. BMC Bioinformatics. 2011;12:116.

26. Edgar RC. MUSCLE: A multiple sequence alignment method with reduced time and space complexity. BMC Bioinformatics. 2004;5:113.

27. Price MN, Dehal PS, Arkin AP. FastTree: computing large minimum evolution trees with profiles instead of a distance matrix. Mol Biol Evol. 2009;26(7):1641-50.

28. Brynildsrud O, Bohlin J, Scheffer L, Eldholm V. Rapid scoring of genes in microbial pan-genome-wide association studies with Scoary. Genome Biol. 2016;17(1):238.

29. McArthur AG, Waglechner N, Nizam F, Yan A, Azad MA, Baylay AJ, Bhullar K Canova MJ, De Pascale G, Ejim L, Kalan L, King AM, Koteva K, Morar M, Mulvey MR, O'Brien JS, Pawlowski AC, Piddock LJ, Spanogiannopoulos P, Sutherland AD, Tang I, Taylor PL, Thaker M, Wang W, Yan M, YU T, Wright $\mathrm{GD}$. The comprehensive antibiotic resistance database. Antimicrob Agents Chemother. 2013;57(7):3348-57.

30. Chen L, Yang J, Yu J, Yao Z, Sun L, Shen Y, Jin Q. VFDB: a reference database for bacterial virulence factors. Nucleic Acids Res. 2005;33(Database issue):D325-8.

31. Arndt D, Grant JR, Marcu A, Sajed T, Pon A, Liang Y, Wishart DS. PHASTER: a better, faster version of the PHAST phage search tool. Nucleic Acids Res. 2016;44(W1):W16-21.

32. Lee EW, Chen J, Huda MN, Kuroda T, Mizushima T, Tsuchiya T. Functional cloning and expression of emeA, and characterization of EmeA, a multidrug efflux pump from Enterococcus faecalis. Biol Pharm Bull. 2003:26(2):266-70.

33. Courvalin P. Vancomycin resistance in gram-positive cocci. Clin Infect Dis. 2006:42(Suppl 1):S25-34

34. Dean M, Hamon Y, Chimini G. The human ATP-binding cassette (ABC) transporter superfamily. J Lipid Res. 2001:42(7):1007-17.

35. Arber W, Linn S. DNA modification and restriction. Annu Rev Biochem. 1969 38:467-500.

36. Kruger DH, Bickle TA. Bacteriophage survival: multiple mechanisms for avoiding the deoxyribonucleic acid restriction systems of their hosts. Microbiol Rev. 1983;47(3):345-60. 
37. Bickle TA, Kruger DH. Biology of DNA restriction. Microbiol Rev. 1993;57(2): 434-50.

38. Murray NE. Type I restriction systems: sophisticated molecular machines (a legacy of Bertani and Weigle). Microbiol Mol Biol Rev. 2000;64(2):412-34.

39. Bonacina J, Suarez N, Hormigo R, Fadda S, Lechner M. Saavedra L. a genomic view of food-related and probiotic Enterococcus strains. DNA Res. 2017;24(1):11-24.

40. Siezen RJ, Renckens B, van Swam I, Peters S, van Kranenburg R, Kleerebezem M, de Vos WM. Complete sequences of four plasmids of Lactococcus lactis subsp. cremoris SK11 reveal extensive adaptation to the dairy environment. Appl Environ Microbiol. 2005;71(12):8371-82.

41. Zeng L, Das S, Burne RA. Utilization of lactose and galactose by Streptococcus mutans: transport, toxicity, and carbon catabolite repression. J Bacteriol. 2010;192(9):2434-44.

42. Rodionov DA, Hebbeln P, Gelfand MS, Eitinger T. Comparative and functional genomic analysis of prokaryotic nickel and cobalt uptake transporters: evidence for a novel group of ATP-binding cassette transporters. J Bacteriol. 2006;188(1):317-27.

43. Palmieri M, Mazur A, Berry GT, Ning C, Wehrli S, Yager C, Reynolds R, Singh R, Muralidharan K, Langley S, Elsas L 2nd, Segal S, Urine S. Plasma galactitol in patients with galactose-1-phosphate uridyltransferase deficiency galactosemia. Metabolism. 1999;48(10):1294-302.

44. Ning C, Segal S. Plasma galactose and galactitol concentration in patients with galactose-1-phosphate uridyltransferase deficiency galactosemia: determination by gas chromatography/mass spectrometry. Metabolism. 2000;49(11):1460-6.

45. Akhtar M, Hirt H, Zurek L. Horizontal transfer of the tetracycline resistance gene tetM mediated by pCF10 among Enterococcus faecalis in the house fly (Musca domestica L.) alimentary canal. Microb Ecol. 2009;58(3):509-18.

46. Poole K. Efflux-mediated antimicrobial resistance. J Antimicrob Chemother. 2005;56(1):20-51.

Ready to submit your research? Choose BMC and benefit from:

- fast, convenient online submission

- thorough peer review by experienced researchers in your field

- rapid publication on acceptance

- support for research data, including large and complex data types

- gold Open Access which fosters wider collaboration and increased citations

- maximum visibility for your research: over $100 \mathrm{M}$ website views per year

At BMC, research is always in progress.

Learn more biomedcentral.com/submissions 